\title{
Sequential treatment of tyrosine kinase inhibitors and chemotherapy for EGFR-mutated non-small cell lung cancer: a meta-analysis of Phase III trials
}

This article was published in the following Dove Press journal:

OncoTargets and Therapy

28 November 2013

Number of times this article has been viewed

\section{Yiliang Zhangl,* \\ Yihua Sun ${ }^{1, *}$ \\ Lei Wang' \\ Ting Yel \\ Yunjian Pan' \\ Haichuan $\mathrm{Hu}^{\prime}$ \\ Yongfu Yu \\ Naiqing Zhao ${ }^{2}$ \\ Yanyan Song ${ }^{3}$ \\ David Garfield ${ }^{4}$ \\ Haiquan Chen'}

'Department of Thoracic Surgery, Fudan University Shanghai Cancer

Center, Department of Oncology,

Shanghai Medical College, Fudan

University, ${ }^{2}$ Department of

Biostatistics, School of Public Health,

Fudan University, ${ }^{3}$ Department of

Pharmacology and Biostatistics,

Institute of Medical Science, Shanghai Jiaotong University School of

Medicine, ${ }^{4}$ ProMed Cancer Centers,

Shanghai, People's Republic of China

*These authors contributed equally to this work

Correspondence: Haiquan Chen Department of Thoracic Surgery, Fudan University Shanghai Cancer Center,

270 Dong An Road, Shanghai 200032,

People's Republic of China

Tel +862164175590

Fax +86 2I 6268 65II

Email hqchen!@yahoo.com
Background: This aim of this study was to compare the efficacy of first-line tyrosine kinase inhibitor therapy followed, upon progression, by chemotherapy with the reverse sequence in patients with EGFR-mutated non-small cell lung cancer (NSCLC) in terms of overall survival.

Methods: We performed a meta-analysis of studies that met the following criteria: Phase III clinical trial comparing the sequencing of epidermal growth factor receptor (EGFR) tyrosine kinase inhibitors with chemotherapy in the treatment of advanced EGFR-mutated NSCLC; activating mutations reported; and availability of hazard ratio estimates with $95 \%$ confidence intervals (CIs) for overall survival.

Results: Six clinical trials were included in this study. The pooled hazard ratio for overall survival of the EGFR-mutated population that completed sequential treatment was 1.03 (95\% CI $0.86-1.22, P=0.776$ ). There was no statistically significant heterogeneity between the studies $\left(\operatorname{tau}^{2}=0 ; \mathrm{I}^{2}=0,95 \% \mathrm{CI} 0-0.37, P=0.548\right)$. Evidence of marked publication bias for the two treatment sequences was insufficient $(P=0.145)$.

Conclusion: In patients with advanced NSCLC and activating EGFR mutations, first-line chemotherapy followed upon progression by a tyrosine kinase inhibitor was not inferior in terms of overall survival compared with the inverse sequence. This may serve as an indication that chemotherapy could be employed initially if mutation testing results are unavailable.

Keywords: EGFR mutation, tyrosine kinase inhibitor, chemotherapy, non-small cell lung cancer, clinical trial

\section{Introduction}

Epidermal growth factor receptor (EGFR) is involved in the development and progression of human non-small cell lung cancer (NSCLC). Erlotinib and gefitinib are oral EGFR tyrosine kinase inhibitors (TKIs) that have been proved effective for patients with advanced NSCLC in whom systemic chemotherapy has failed or as first-line therapy. ${ }^{1-4}$ Further studies have demonstrated that the efficacy of EGFR TKIs is greatest in the subset of patients with NSCLC who harbor somatic mutations in the EGFR kinase domain, ${ }^{5}$ and this has been confirmed in prospective clinical trials. ${ }^{6}$ Based on these data, gefitinib was initially approved by the US Food and Drug Administration as first-line treatment for EGFR-mutated NSCLC.

The OPTIMAL (Erlotinib versus chemotherapy as first-line treatment for patients with advanced EGFR mutation-positive non-small-cell lung cancer) study presented its updated overall survival data at the 2012 American Society of Clinical Oncology conference, showing that patients with EGFR-mutated NSCLC appeared to benefit more 
from the sequential combination of TKIs and chemotherapy than from either treatment alone. ${ }^{7}$ The Phase III TORCH (Tarceva or Chemotherapy) study compared first-line erlotinib, followed by second-line cisplatin/gemcitabine, with the reverse treatment sequence. This study concluded that the former was significantly inferior in terms of overall survival compared with the latter. ${ }^{8}$ However, whether a TKI or chemotherapy should be first-line in patients with activating EGFR mutations is still unknown. Therefore, we performed a meta-analysis of the recent Phase III trials which compared overall survival on first-line TKIs (erlotinib or gefitinib) followed at progression by chemotherapy (TKIChemo) over the reverse treatment (Chemo-TKI) in patients with EGFR-mutant NSCLC.

\section{Materials and methods}

\section{Search strategy and eligibility criteria}

The literature search was conducted up to July 31, 2013 in the PubMed and Embase databases using the search terms "lung cancer" and "erlotinib or gefitinib" and limiting the results to Phase III clinical trials and English publications. Results from the initial search were subsequently handscreened for eligibility. Studies were included in the systemic review if they met the following criteria: Phase III clinical trial comparing the sequencing of EGFR TKIs (erlotinib or gefitinib) with chemotherapy in the treatment of advanced EGFR-mutated NSCLC; activating mutations reported; and hazard ratio estimates with $95 \%$ confidence intervals (CIs, or data to calculate these) for overall survival available for the EGFR-mutated population. Abstracts presented at major oncologic meetings were also searched for unpublished data. Only the most recent information was included where data sets overlapped or were duplicated.

\section{Data extraction}

Subgroup information was manually extracted from eligible studies. This was carried out independently by two authors (YZ and LW) and cross-checked to reach consensus. The following variables were recorded: trial name, publication year, country or region where the study was performed, sample size of patients with EGFR-mutated NSCLC who received crossover therapies (TKI-Chemo or Chemo-TKI), median overall survival, and hazard ratio estimates with corresponding 95\% CIs for overall survival of the intent-to-treat population. The hazard ratio and its variance were estimated and extracted using a previously reported approach. ${ }^{9}$ If necessary, the primary authors were contacted to retrieve missing data.

\section{Statistical analysis}

Overall log hazard ratios and 95\% CIs for overall survival were estimated using already published methods ${ }^{10}$ and reported from analyses using random effects models. Between-study heterogeneity was evaluated by tau ${ }^{2}$ and $\mathrm{I}^{2}$ indices using previously reported methods. ${ }^{11}$ Sensitivity analysis was performed to investigate the influence of a single study on the overall meta-analysis estimate. Because there is currently no approach reliable enough to indicate publication bias when the number of the studies is small, ${ }^{12}$ the Egger test was used only for exclusion of a potentially distinct bias. All $P$-values were two-sided and all statistical tests were performed using Stata version 12.0 software (Stata Corporation, College Station, TX, USA). This systematic review was planned, conducted, and reported in adherence with the standards of quality for reporting meta-analyses. ${ }^{13}$

\section{Results}

\section{Literature search}

A flow diagram of the literature search is shown in Figure 1. Six clinical trials were finally included, ie, IPASS (the Iressa Pan-Asia Study) ${ }^{4,14}$ NEJ002, ${ }^{6,15}$ WJTOG3405, ${ }^{16}$ OPTIMAL, ${ }^{7,17}$ First-SIGNAL (first-line single-agent Iressa versus gemcitabine and cisplatin trial in never-smokers with adenocarcinoma of the lung), ${ }^{18}$ and TORCH. ${ }^{8}$ These were all multicenter, open-label, Phase III trials which were similarly designed and enrolled patients with well matched baseline characteristics. Notable exceptions included NEJ002, WJTOG3405, and OPTIMAL, in which the study population had known mutated EGFR tumors, while the other three studies (IPASS, First-SIGNAL, and TORCH) conducted EGFR mutation testing in qualifying samples after the trial launch. EURTAC (European Randomized Trial of Tarceva Versus Chemotherapy) ${ }^{3}$ was not included because overall survival data for the target patients were unavailable.

\section{Study characteristics}

The trials on first-line use of TKIs were carried out between 2005 and 2009 and involved a total of 2,635 patients who were chemotherapy-naive before enrolment. Of these six studies, two were conducted in Japan and three were done in Korea, the People's Republic of China, and South-East Asia. TORCH, however, was performed in Europe and North America. Activating EGFR mutations were determined before or during the studies, and the qualifying mutational types were deletion in exon 19 and the L858R mutation in exon 21, both of which are deemed sensitive to EGFR TKIs. Three trials (NEJ002, WJOTG3405, OPTIMAL) restricted 


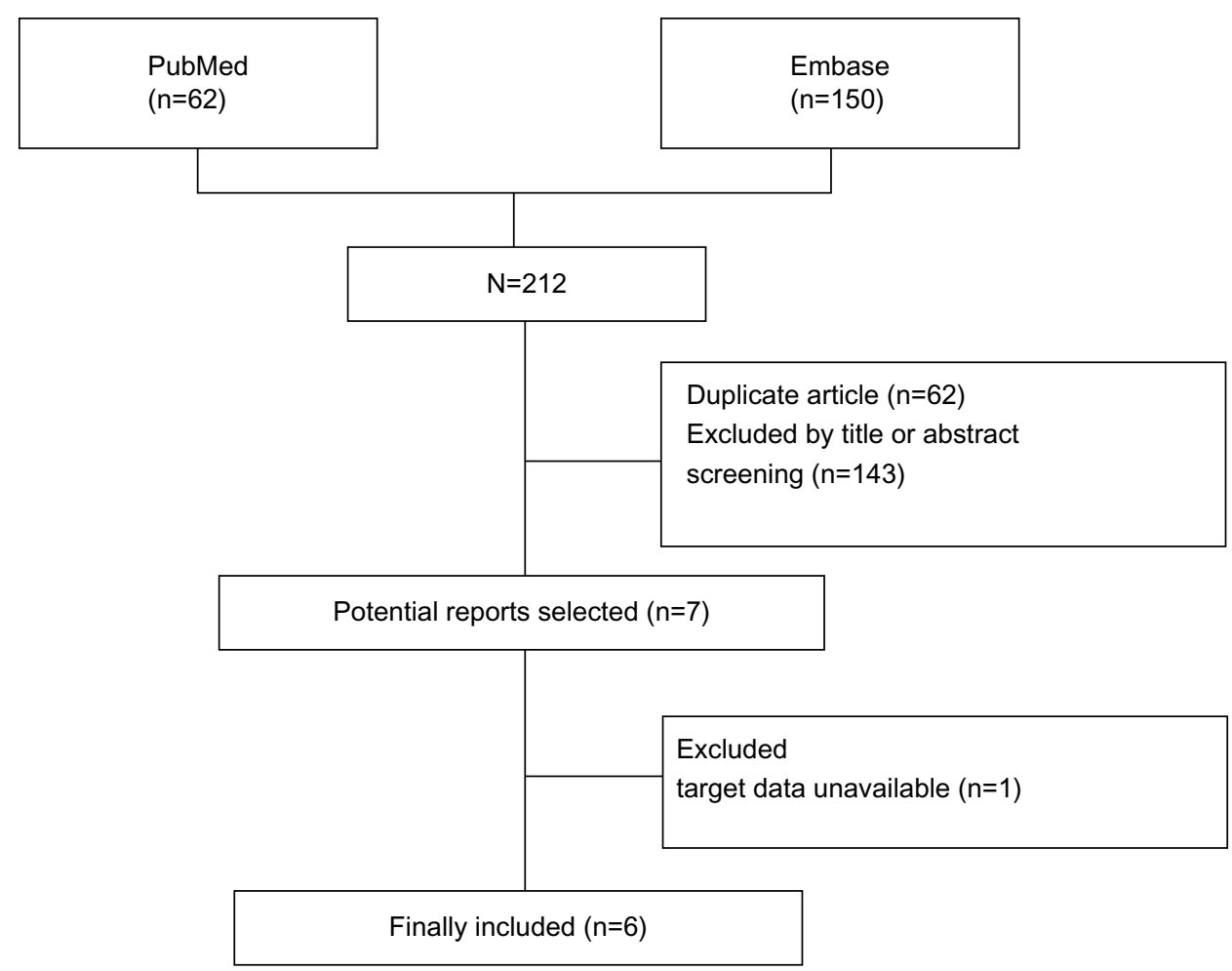

Figure I Study flow chart showing process for selecting eligible publications.

enrolment to the activating EGFR-mutated population. There were two studies (IPASS and First-SIGNAL) that enrolled patients with clinical characteristics (East Asians with pulmonary adenocarcinoma who were nonsmokers or former light smokers) known to be associated with these activating mutations. In the TORCH study, clinical or biologic factors were not applied in the selection of the study population. Mutation analysis performed on available tumor samples revealed that the EGFR-mutated population was 261 (59.7\%) in IPASS, 42 (44\%) in First-SIGNAL, and 39 (14.2\%) in TORCH. Patients were randomized to receive either firstline TKIs (gefitinib $250 \mathrm{mg}$ or erlotinib $150 \mathrm{mg}$ daily) or platinum-based chemotherapy for 3-9 cycles as tolerated or until disease progression.
After disease progression, all patients in the TORCH trial entered second-line crossover treatments of the same regimens and doses as initially designed. In the other five studies, the strategies for second-line methods were not uniform. However, many patients $(76.2 \%$ in all) chose to receive the crossover treatment, the same as the opposite arm designed in each trial. Additionally, there was a higher crossover proportion in the first-line chemotherapy arm than in the TKI arm (68.6\% versus $80.5 \%, P=0.045$, Table 1).

\section{Overall survival}

None of the studies showed a significant overall survival benefit for first-line EGFR TKIs followed by

Table I Characteristics of the included studies

\begin{tabular}{|c|c|c|c|c|c|c|c|c|c|}
\hline \multirow[t]{2}{*}{ Study } & \multirow[t]{2}{*}{ Region } & \multirow{2}{*}{$\begin{array}{l}\text { Overall } \\
\text { patients (n) }\end{array}$} & \multirow{2}{*}{$\begin{array}{l}\text { EGFR mut + } \\
\text { patients }\end{array}$} & \multicolumn{3}{|c|}{ TKI-Chemo arm } & \multicolumn{3}{|c|}{ Chemo-TKI arm } \\
\hline & & & & $\begin{array}{l}\text { Crossover } \\
\text { rate (\%) }\end{array}$ & $\begin{array}{l}\text { Patients } \\
\text { (n) }\end{array}$ & $\begin{array}{l}\text { Median OS } \\
\text { (months) }\end{array}$ & $\begin{array}{l}\text { Crossover } \\
\text { rate (\%) }\end{array}$ & $\begin{array}{l}\text { Patients } \\
\text { (n) }\end{array}$ & $\begin{array}{l}\text { Median OS } \\
\text { (months) }\end{array}$ \\
\hline TORCH & Italy/Canada & 760 & 39 & 58.3 & 19 & 18.1 & 71.5 & 20 & 32.5 \\
\hline First-SIGNAL & Korea & 313 & 42 & NA & 26 & 27.2 & 75.0 & 16 & 25.6 \\
\hline IPASS & South Asia & 1,217 & 261 & 75.0 & 99 & 21.6 & 64.3 & 83 & 21.9 \\
\hline NEJ002 & Japan & 228 & 228 & 66.7 & 76 & 27.7 & 98.0 & 112 & 26.6 \\
\hline WJTOG3405 & Japan & 172 & 172 & 69.8 & 60 & 35.5 & 90.7 & 78 & 38.8 \\
\hline OPTIMAL & Chinese & 154 & 154 & 52.0 & 43 & 30.4 & 71.0 & 51 & 31.5 \\
\hline
\end{tabular}

Abbreviations: mut, mutation; TKI, tyrosine kinase inhibitor; Chemo, chemotherapy; OS, overall survival; $\mathrm{HR}$, hazard ratio; $\mathrm{Cl}$, confidence interval. 
second-line chemotherapy versus the reverse sequence in the EGFR-mutated population. However, the TORCH trial appeared to demonstrate a preference for the Chemo-TKI strategy. Median overall survival for the EGFR mutation subgroup was 18.1 months versus 32.5 months (hazard ratio 1.58; 95\% CI 0.70-3.57). ${ }^{8}$ In the OPTIMAL trial, conducted in a Chinese population, the two sequential treatments were nearly identical, with a median overall survival of 30.4 (TKIChemo arm) versus 31.5 months (Chemo-TKI arm) and a hazard ratio of $1.08(95 \%$ CI $0.61-1.91) .^{5}$

The other four trials (IPASS, NEJ002, WJTOG3405, and First-SIGNAL) did not contain overall survival data (survival curve, median overall survival, or hazard ratio) for EGFR-mutated patients receiving crossover treatments. However, they did provide Kaplan-Meier curves and hazard ratios for those with EGFR mutation-positive NSCLC. Due to the high proportion of crossover patients at second-line treatment $(76.9 \%$ on average for each trial), the hazard ratio and its 95\% CI for overall survival of all EGFR-mutated patients could be estimated as the hazard ratio of the target population. The estimated hazard ratio for overall survival was 1.00 (95\% CI 0.76-1.33) for IPASS, 0.89 (95\% CI 0.63-1.24) for NEJ002, 1.18 (95\% CI 0.77-1.83) for WJTOG3405, and 1.04 (95\% CI 0.50-2.18) for First-SIGNAL. The pooled hazard ratio for overall survival of the EGFR-mutated population completing sequential treatments was $1.03(95 \%$ CI $0.86-1.22, P=0.776$, Figure 2). Sensitivity analysis showed that in no study was there a significant influence on the overall result (Supplementary Figure 1). This implies that for patients with mutated NSCLC (common deletions in exon 19 or L858R in exon 21), first-line chemotherapy followed at progression by EGFR TKI therapy was not inferior in terms of overall survival compared with the inverse sequence of first-line TKI followed by chemotherapy. The estimated indices indicate that there was no statistically significant heterogeneity among the studies $\left(\operatorname{tau}^{2}=0, \mathrm{I}^{2}=0,95 \% \mathrm{CI} 0-0.37, P=0.548\right)$. The Egger test had a $P$-value of 0.145 , suggesting that evidence of marked publication bias for the two treatment sequencings was insufficient (Supplementary Figure 2).

\section{Discussion}

Erlotinib and gefitinib were the first EGFR TKIs approved for the treatment of advanced NSCLC. ${ }^{19}$ Data from clinical trials have provided good evidence that these agents can be used as first-line therapy in patients with tumors harboring activating EGFR mutations. Moreover, the OPTIMAL study presented at the 2012 American Society of Clinical Oncology annual meeting showed that patients with EGFR-mutated NSCLC appeared to benefit more from the sequential combination of a TKI and chemotherapy than from either treatment alone. ${ }^{5}$ Also, the Phase III TORCH study compared first-line erlotinib followed at progression by standard chemotherapy versus the reverse sequence. TORCH concluded that in unselected patients with advanced NSCLC, first-line erlotinib followed at progression by chemotherapy was significantly inferior in terms of overall survival compared with the standard

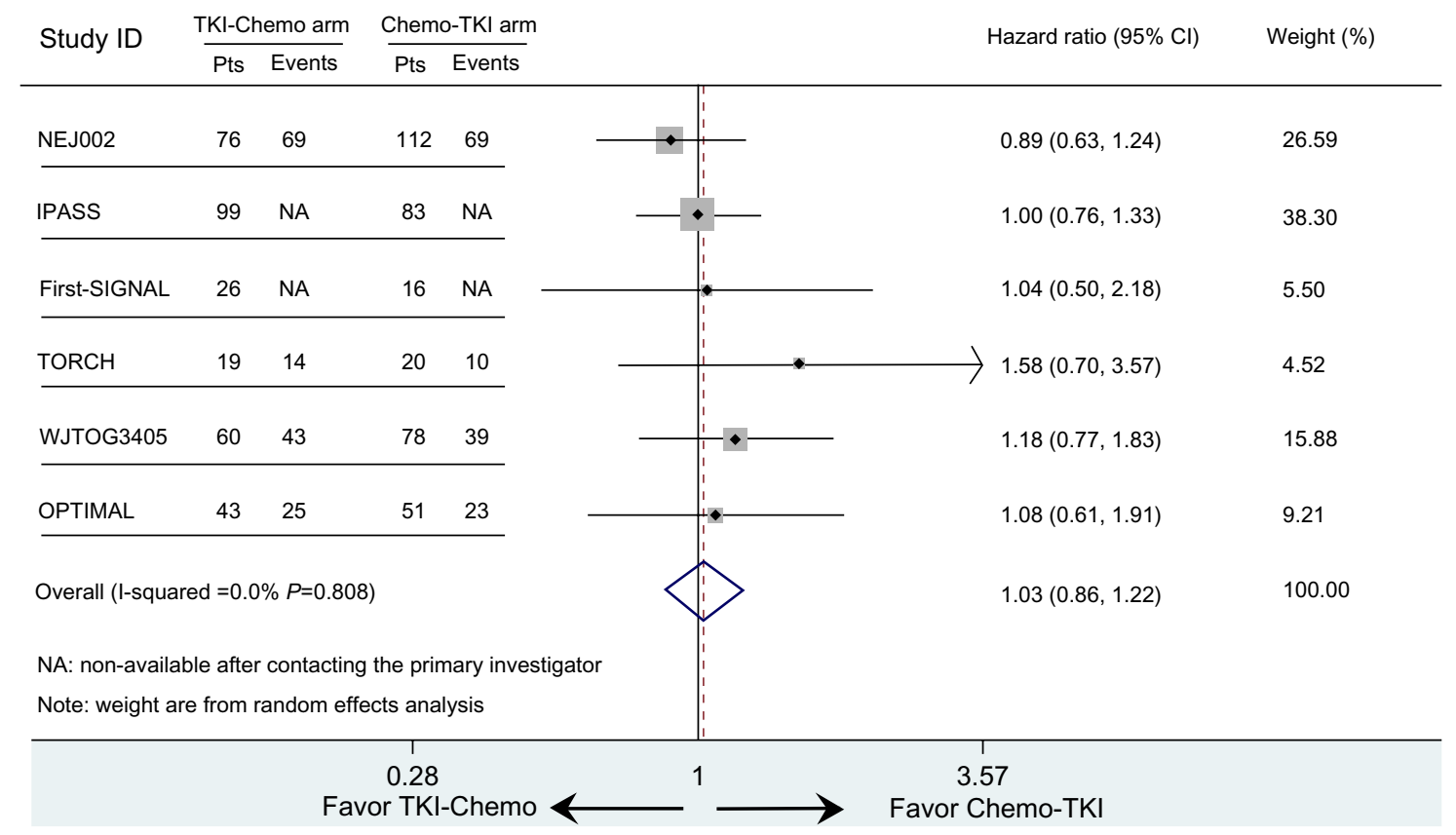

Figure 2 Forest plot of overall survival in patients with activating EGFR-mutated non-small cell lung cancer after crossover treatment. Abbreviations: TKI, tyrosine kinase inhibitor; $\mathrm{Cl}$, confidence interval; Chemo, chemotherapy. 
sequence of first-line chemotherapy followed by erlotinib. However, for patients with activating EGFR mutations, the better sequence remains undetermined. Thus, we performed this systematic review in an attempt to identify and quantify any overall survival benefits of sequential therapy of TKI and chemotherapy in patients with advanced NSCLC and activating EGFR mutations. Based on the enrolled studies, the pooled hazard ratio for overall survival demonstrated no significant difference between the sequencings. We also confirmed the overall survival results from individual trials, in which most patients received and benefited from crossover treatment at progression. Our results also suggest that, in patients with NSCLC and EGFR mutations, first-line chemotherapy followed at progression by EGFR TKI therapy is not inferior in terms of overall survival compared with the inverse sequence of first-line TKI followed by chemotherapy. Therefore, we suggest that chemotherapy can be used in advance of mutation testing results if they are not immediately available for whatever reason.

Concern can be raised regarding the rate of crossover to EGFR TKI therapy after first-line chemotherapy, given that a considerable number of patients (up to $30 \%$ ) assigned to first-line chemotherapy did not switch to EGFR TKI therapy. Limited information was available in these trials, except in TORCH, which noted that 90 patients $(28.5 \%)$ did not receive second-line erlotinib, mainly because of worsening condition or death (56 cases, 62.2\%) and other reasons, such as patients choosing other treatments (15 cases, 16.7\%) or refusal (seven cases, $7.8 \%$ ).

Meta-analysis is an important tool for revealing trends that might not be apparent in a single study, and pooling of independent but similar studies increases precision and therefore the level of confidence in the findings. ${ }^{13}$ The current meta-analysis has advantages. First, our quantitative assessments were based on subsamples retrieved from well known multicenter, open-label, randomized, controlled Phase III trials, thus minimizing the possibility of betweenstudy variance resulting from recall or selection bias. Second, the total number of cases and controls was substantial and analyzed using the intent-to-treat method, thus significantly increasing the statistical power of the analysis.

Despite these advantages, some limitations should be acknowledged. First, four of the six studies (IPASS, NEJ002, WJTOG3405, First-SIGNAL) only stratified overall survival data for the EGFR-mutated population but went no further for those receiving crossover treatment. The hazard ratio for overall survival was estimated as that for patients with EGFR-mutated NSCLC, including a few patients
(23.2\% in total) who did not cross over. This likely exerts some bias. However, bias may not impact the final result for the pooled hazard ratio. On the one hand, 64.3\%-98\% of all patients in each arm received reverse therapy at progression in the eligible studies (Table 1), and the OPTIMAL data have proved that these people achieved better survival than those who did not. ${ }^{7}$ On the other hand, almost all the relapsed patients received crossover treatment and contributed the most weight in the survival curve. As a result, the hazard ratio for overall survival would be similar between $E G F R$-mutant patients who received crossover therapy and the entire $E G F R$-mutant population. The best evidence for this comes from the OPTIMAL study, in which hazard ratios for the two subgroups were provided separately and were similar to each other, ie, 1.08 (95\% CI 0.61-1.91) for the EGFR-mutated patients who had a crossover strategy versus 1.07 (95\% CI 0.69-1.58) for the whole EGFR-mutant population. Second, there is no method reliable enough to indicate publication bias when study numbers are small. ${ }^{12} \mathrm{We}$ used the Egger test here only to exclude potentially marked bias. However, for this systematic review, all included trials were published with nonsignificant results. If there was one study with positive results, it should have been published. Thus, evidence of marked publication bias appears to be insufficient. Third, other factors, such as toxicity, quality of life/symptom reduction, and progression-free survival, which are clinically relevant when making a treatment decision, were not discussed in this paper. It is almost impossible to undertake a meta-analysis on those factors with the existing data because individual patient data were not obtained for practical reasons. Finally, crossover therapy after failure of first-line treatment was not randomized in five of the six trials (only TORCH had a crossover strategy design). Thus, the crossover procedure in these five studies was not in random but in observational fashion, which may lead to heterogeneity between studies. We further performed a sensitivity analysis, showing that the results of the studies were consistent with and without TORCH. Therefore, the final conclusion was robust and reliable (Supplementary Figure 1).

This systematic review of the sequencing of EGFR TKI with chemotherapy implies that, in the treatment of advanced EGFR-mutated NSCLC (common mutations in exon 19 and L858R in exon 21), first-line chemotherapy followed at progression by an EGFR TKI was not inferior in terms of overall survival compared with the inverse sequence of first-line TKI followed by chemotherapy. Importantly, this indicates that chemotherapy could be employed initially if mutation testing results are not immediately available. Nevertheless, further 
prospective clinical trials are needed for more substantial evidence to clarify this issue.

\section{Disclosure}

The authors report no conflicts of interest in this work.

\section{References}

1. Kim ES, Hirsh V, Mok T, et al. Gefitinib versus docetaxel in previously treated non-small-cell lung cancer (INTEREST): a randomised phase III trial. Lancet. 2008;372:1809-1818.

2. Shepherd FA, Rodrigues PJ, Ciuleanu T, et al. Erlotinib in previously treated non-small-cell lung cancer. $N$ Engl J Med. 2005;353:123-132.

3. Rosell R, Carcereny E, Gervais R, et al. Erlotinib versus standard chemotherapy as first-line treatment for European patients with advanced EGFR mutation-positive non-small-cell lung cancer (EURTAC): a multicentre, open-label, randomised phase 3 trial. Lancet Oncol. 2010;13:239-246.

4. Mok TS, Wu YL, Thongprasert S, et al. Gefitinib or carboplatin-paclitaxel in pulmonary adenocarcinoma. N Engl J Med. 2009;361:947-957.

5. Paez JG, Janne PA, Lee JC, et al. EGFR mutations in lung cancer: correlation with clinical response to gefitinib therapy. Science. 2004;304: 1497-1500.

6. Maemondo M, Inoue A, Kobayashi K, et al. Gefitinib or chemotherapy for non-small-cell lung cancer with mutated EGFR. $N$ Engl $J$ Med. 2010;362:2380-2388.

7. Zhou C, Wu Y, Liu X, et al. Overall survival (OS) results from OPTIMAL (CTONG0802), a phase III trial of erlotinib (E) versus carboplatin plus gemcitabine (GC) as first-line treatment for Chinese patients with EGFR mutation-positive advanced non-small cell lung cancer (NSCLC). $J$ Clin Oncol. 2012;30 Suppl:Abstr 7520.

8. Gridelli C, Ciardiello F, Gallo C, et al. First-line erlotinib followed by second-line cisplatin-gemcitabine chemotherapy in advanced non-smallcell lung cancer: the TORCH randomized trial. J Clin Oncol. 2011;30: 3002-3011.

9. Parmar MK, Torri V, Stewart L. Extracting summary statistics to perform meta-analyses of the published literature for survival endpoints. Stat Med. 1998;17:2815-2834.
10. Tierney JF, Stewart LA, Ghersi D, Burdett S, Sydes MR. Practical methods for incorporating summary time-to-event data into metaanalysis. Trials. 2007;8:16.

11. Huedo-Medina TB, Sanchez-Meca J, Marin-Martinez F, Botella J. Assessing heterogeneity in meta-analysis: Q statistic or I2 index? Psychol Methods. 2006;11:193-206.

12. Ahmed I, Sutton AJ, Riley RD. Assessment of publication bias, selection bias, and unavailable data in meta-analyses using individual participant data: a database survey. BMJ. 2012;344:d7762.

13. Stroup DF, Berlin JA, Morton SC, et al. Meta-analysis of observational studies in epidemiology: a proposal for reporting. Meta-analysis Of Observational Studies in Epidemiology (MOOSE) group. JAMA. 2000;283:2008-2012.

14. Fukuoka M, Wu YL, Thongprasert S, et al. Biomarker analyses and final overall survival results from a phase III, randomized, open-label, first-line study of gefitinib versus carboplatin/paclitaxel in clinically selected patients with advanced non-small-cell lung cancer in Asia (IPASS). J Clin Oncol. 2011;29:2866-2874.

15. Inoue A, Kobayashi K, Maemondo M, et al. Updated overall survival results from a randomized phase III trial comparing gefitinib with carboplatin-paclitaxel for chemo-naive non-small cell lung cancer with sensitive EGFR gene mutations (NEJ002). Ann Oncol. 2013;24:54-59.

16. Mitsudomi T, Morita S, Yatabe Y, et al. Gefitinib versus cisplatin plus docetaxel in patients with non-small-cell lung cancer harbouring mutations of the epidermal growth factor receptor (WJTOG3405): an open label, randomised phase 3 trial. Lancet Oncol. 2010;11:121-128.

17. Zhou C, Wu YL, Chen G, et al. Erlotinib versus chemotherapy as first-line treatment for patients with advanced EGFR mutationpositive non-small-cell lung cancer (OPTIMAL, CTONG-0802): a multicentre, open-label, randomised, phase 3 study. Lancet Oncol. 2011;12:735-742.

18. Han JY, Park K, Kim SW, et al. First-SIGNAL: first-line single-agent Iressa versus gemcitabine and cisplatin trial in never-smokers with adenocarcinoma of the lung. J Clin Oncol. 2012;30:1122-1128.

19. Ciardiello F, Tortora G. EGFR antagonists in cancer treatment. $N$ Engl J Med. 2008;358:1160-1174. 


\section{Supplementary figures}

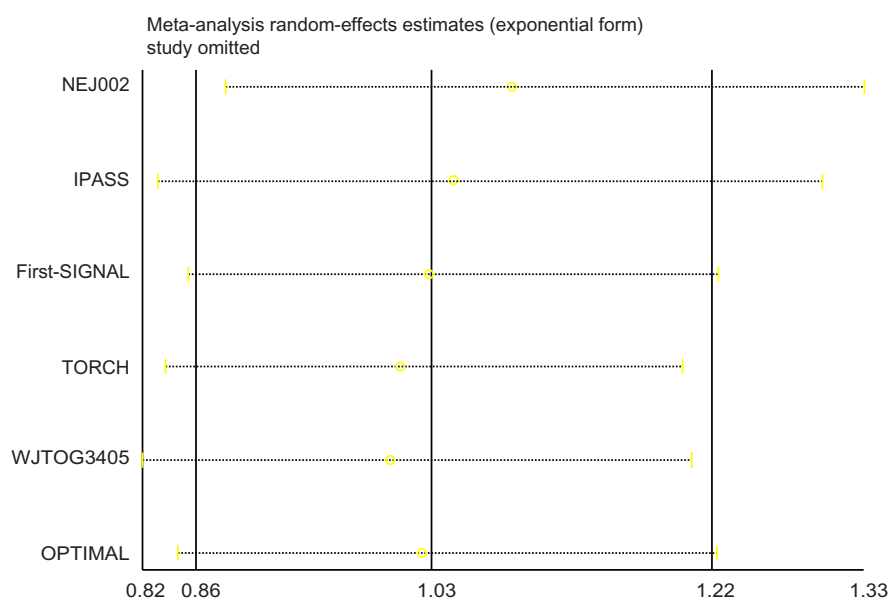

Figure SI Sensitivity analysis demonstrated no study exerting significant influence on overall result.

Egger's publication bias plot

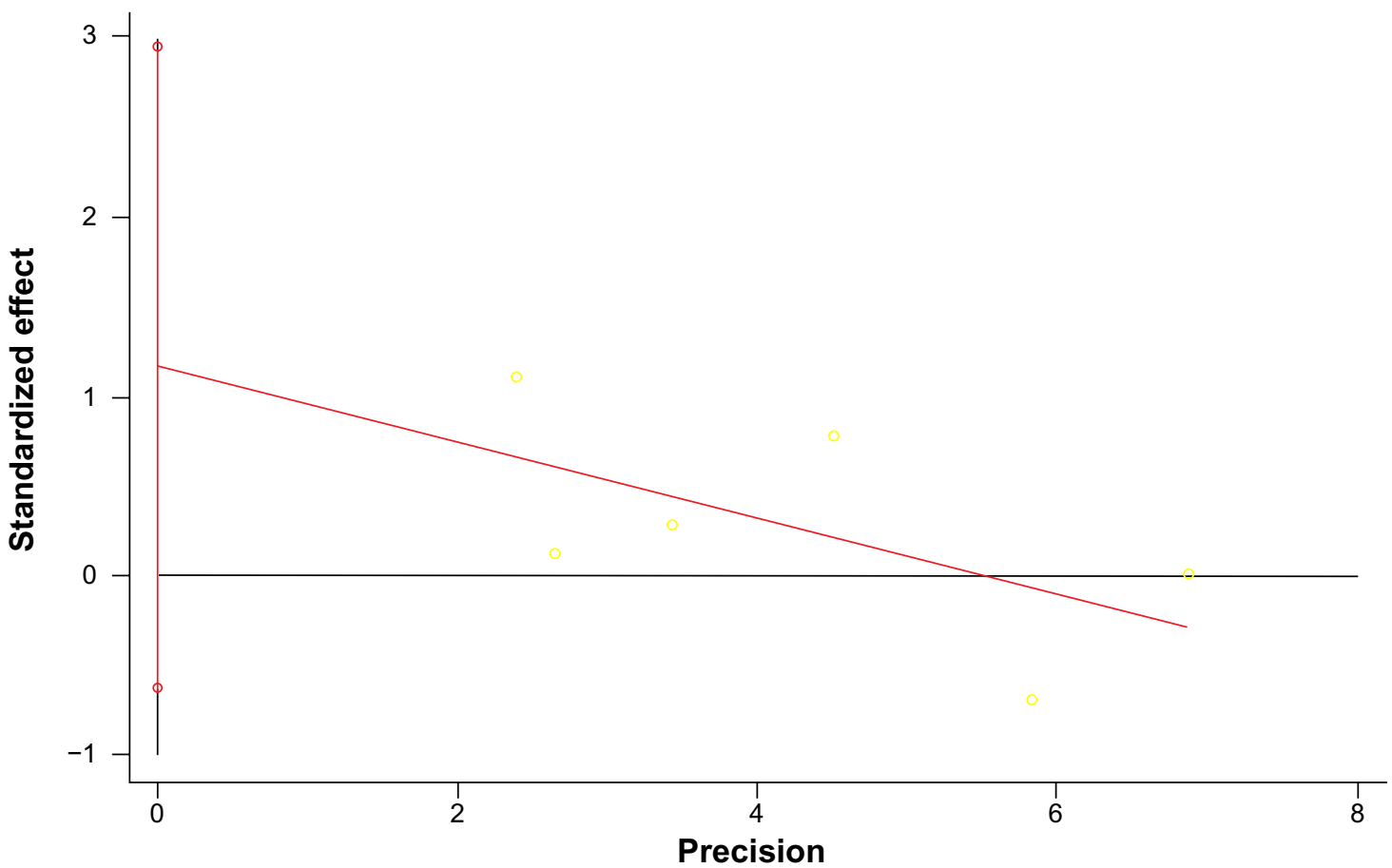

Figure S2 Publication bias plot based on the Egger test.

\section{Publish your work in this journal}

OncoTargets and Therapy is an international, peer-reviewed, open access journal focusing on the pathological basis of all cancers, potential targets for therapy and treatment protocols employed to improve the management of cancer patients. The journal also focuses on the impact of management programs and new therapeutic agents and protocols on patient perspectives such as quality of life, adherence and satisfaction. The manuscript management system is completely online and includes a very quick and fair peer-review system, which is all easy to use. Visit http://www.dovepress.com/testimonials.php to read real quotes from published authors.

Submit your manuscript here: http://www.dovepress.com/oncotargets-and-therapy-journal 